\title{
Severe Hypertriglyceridemia Presenting as Superior Sagittal Sinus Thrombosis
}

\author{
Debmalya Sanyal, Soumyabrata Roy Chaudhuri, Anirban Majumder \\ Department of Endocrinology, Kali Pradip Chaudhari Medical College, Kolkata, India
}

\begin{abstract}
We report a case of a 38-year-old Indian male who presented with severe throbbing headache spreading diffusely from occipital to nuchal regions. He was detected to have superior cerebral (sagittal) venous sinus thrombosis (CVST) with severe hypertriglyceridemia without any coagulation abnormalities or autoimmune disease. Our case highlights the need for clinicians to consider CVST among patients with uncontrolled hypertriglyceridemia. New prognostic measures may become necessary having an implication on the risk of recurrence and duration of anticoagulant therapy in CVST.
\end{abstract}

Key words: hypertriglyceridemia, venous thrombosis, headache

\section{INTRODUCTION}

Cerebral venous sinus thrombosis (CVST) is an uncommon condition with a variable clinical presentation and numerous risk factors. We present a case of superior sagittal sinus thrombosis caused by elevated triglyceridemia. To our knowledge, this is the first report of a CVST in association with hypertriglyceridemia in an adult but in the absence of diabetes with concomitant ketoacidosis or any known pro-thrombotic conditions.

\section{CASE REPORT}

A 38-year-old Indian male was admitted with a two-week history of progressively worsening severe throbbing headache spreading diffusely from occipital to nuchal regions. It was not accompanied by fever, vomiting, nor seizure. A week later, he started having mild blurring of vision and intermittent binocular diplopia. Lipid profile done one month prior to admission showed elevated triglyceride levels of $630 \mathrm{mg} / \mathrm{dl}$ for which no medical treatment was given. On admission, his blood pressure was 150/100 mmHg. Other anthropometric measurements included waist circumference and body mass index, which were within the normal limits (Table 1). Neurological examination revealed bilateral papilledema with left lateral rectus weakness probably due to raised intracranial pressure. There were no meningeal signs, no long tract signs (indicating no involvement of the posterior column, spinothalamic and pyramidal tract) and systemic examination was normal.
Table 1. Anthropometric Measurements and Blood profile

\begin{tabular}{lll}
\multicolumn{1}{c}{ Test } & \multicolumn{1}{c}{ Results } & \multicolumn{1}{c}{ Normal } \\
\hline Haemoglobin, gm/dL & 15.2 & $12-16$ \\
Packed cell volume, \% & 46.5 & $36-46$ \\
Erythrocyte Sedimentation Rate & 15 & $0-22$ \\
Total Leucocyte Count, & 10,000 & $4000-11,000$ \\
(leucocytes/cumm) & & \\
Platelet Count & $250^{*} 10^{\wedge} 3 / \mu \mathrm{L}$ & $150-450^{\star} 10^{\wedge} 3 / \mu \mathrm{L}$ \\
Waist Circumference, cm & 84 & $<90$ \\
Body Mass Index, kg/m ${ }^{2}$ & 21.8 & $18-22.9$ \\
Fasting Plasma Glucose, mg/dL & 92 & $<100$ \\
Glycated Haemoglobin, \% & 5.2 & $<5.7$ \\
Post Prandial Plasma Glucose, & 116 & $<140$ \\
mg/dL & & \\
\hline
\end{tabular}

The patient's hemoglobin, total leucocyte count, ESR and platelet count were within normal ranges (Table 1). Additional laboratory studies showed normal blood chemistry results including electrolytes, renal and liver function tests, FBS and HbA1c (Table 1). The 2D echocardiogram was also normal. His lipid profile showed grossly elevated triglyceride levels of $838 \mathrm{mg} / \mathrm{dl}$ with LDLcholesterol of 120mg/dl and HDL-cholesterol of $48 \mathrm{mg} / \mathrm{dl}$ (Table 2). Cranial computed tomography scan was normal, but magnetic resonance imaging with magnetic resonance angiography of the brain showed thrombosis of the superior sagittal sinus (Figure 1).

\begin{tabular}{lll} 
Table 2. Lipid Profile Test & & \\
\hline \multicolumn{1}{c}{ Test } & Result & Normal \\
\hline Triglyceride,mg/dL & 838 & $<200$ \\
Low Density Lipoprotein Cholesterol, mg/dL & 120 & $<130$ \\
High Density Lipoprotein Cholesterol,mg/dL & 48 & $>40$ \\
\hline
\end{tabular}

Corresponding author: Debmalya Sanyal, $M D$

Associate Professor, Department of Endocrinology

Kali Pradip Chaudhari Medical College

1F, Raja Subodh Chandra Mullick Road, Jadavpur

Kolkata 700032 West Bengal, India

Tel. No.: +33-3001-6100

Fax No.: +33-2418-7459

E-mail:drdebmalyasanyal@gmail.com 


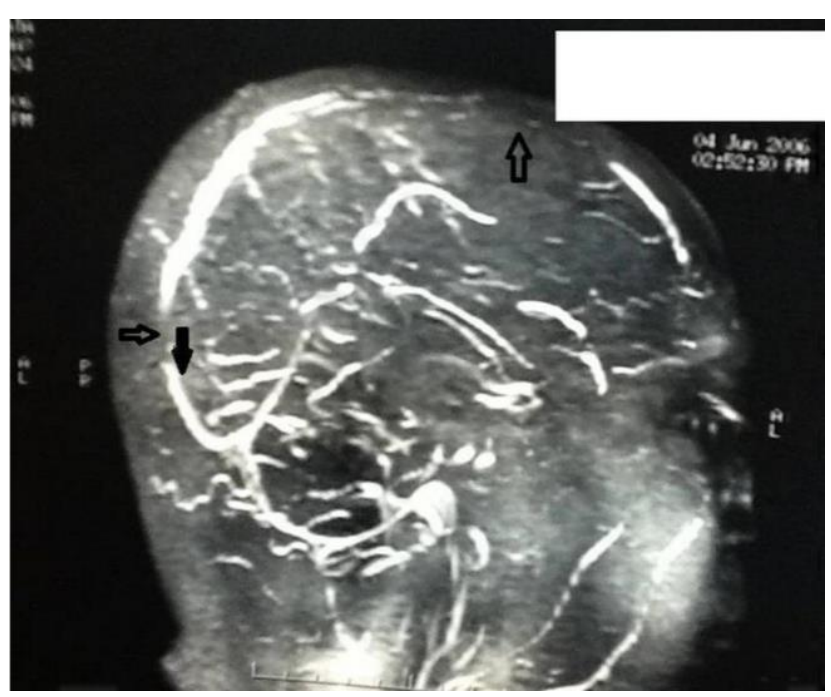

Figure 1. Magnetic resonance imaging (MRI) with magnetic resonance angiography (MRA) brain. The arrow showing thrombosis of superior sagittal sinus thrombosis

Screening for coagulopathies and autoimmune disorders was normal with normal antithrombin III, protein $C$, protein $S$, homocysteine, activated protein $C$, and human factor V. Lupus anticoagulant, anti-cardiolipin antibodies and antinuclear antibodies were negative. He had no history of diabetes, hypertension, nor cancer. He did not smoke and had no history of alcohol intake. He had no similar family history and was not taking any medications.

The patient was put on heparin infusion at 1000 units/hour which was adjusted as per activated partial thromboplastin time. Heparin infusion was continued for eight days. He was discharged on an oral anticoagulant with the measured international normalized ratio (INR) between 2.5 to 3.0. The patient was doing well, his headache subsided and there were no seizures. His lateral rectus palsy had also resolved. In the absence of other predisposing factors, the severe hypertriglyceridemia may have triggered the CVST.

\section{DISCUSSION}

CVST is a relatively rare disorder with an estimated annual incidence of three to four cases per million. ${ }^{1}$ Headache is one of the presenting symptoms in $70-90 \%$ of the cases, while other symptoms include seizures, focal deficits, cranial nerve syndromes involving IX, X, XI or III, IV and VI cranial nerve palsies, visual disturbances, altered consciousness, papilloedema. . $^{2,3}$

The predisposing factors can be identified in only $65-80 \%$ of patients with more than one factor being present in a single patient. These include local and systemic predisposing factors such as pregnancy and puerpuerium, use of oral contraceptive pills, malignancy, connective tissue and inflammatory diseases, increased body mass index, hyperglycaemia, hypertriglyceridemia, haematological conditions and drugs. ${ }^{2,4}$
In our case, hypertriglyceridemia was the only predisposing risk factor-for developing CVST. Dyslipidaemia is associated with significantly increased risk of thrombosis in younger patients who develop venous thromboembolism (VTE) (OR: 2.13; 95\% CI: 1.084.18; $\mathrm{p}=0.0266$ ) but not in older patients. ${ }^{2}$ Meta-analysis of eleven studies ( 8 case-control and 3 cohort studies) by Angeno et al found that patients with VTE had higher triglyceride levels than that of the control population, with a mean difference of $21.0 \mathrm{mg} / \mathrm{dL}$ (95\% CI, 11.0 to $31.0)$ in the case-control studies and $8.6 \mathrm{mg} / \mathrm{dL}$ (95\% CI, 1.2 to 16.0) in the cohort studies. Patients with alterations in the lipid profile had a higher risk of VTE (OR: 1.62, 95\% CI: $1.04-2.52, p=0.03){ }^{9}$

Wang et al., reported the case of a child suffering from acute lymphoblastic leukaemia which illustrated the strong correlation of the rare thrombotic complication, superior sagittal sinus thrombosis and hypercoagulable status secondary to combination use of L-asparaginase and corticosteroid. ${ }^{5}$ The mean peak triglyceride level was significantly higher than the level before therapy. Thus it provides a plausible relationship between elevated triglyceridemia and superior sagittal sinus thrombosis (SSST). Doggen and colleagues conducted a case-control study in post-menopausal women and concluded that elevated triglyceride levels were associated with a doubling of venous thrombosis risk. ${ }^{6}$ Candelaria and associates demonstrated that the odds ratio for the occurrence of deep vein thrombosis in patients with triglyceride levels $\geq 300 \mathrm{mg} / \mathrm{dL}$ was $3.14 .^{7}$ Their results were supported by another study conducted in India which had found a statistically positive correlation between hypercoagulability in nephrotic syndrome, proteinuria, and increased lipid levels. In their paper, they stated that hyperlipidaemia alters the platelet membrane, promoting platelet aggregation. ${ }^{8}$

Mertens and associates have found a statistically significant increased level of leucocyte count and plasminogen activator inhibitor- 1 activity in subjects with metabolic syndrome, defined according to the National Cholesterol Education Program-Adult Treatment Panel III (NCEP-ATP III) criteria or the World Health Organisation criteria. ${ }^{13}$ In our case, the patient did not qualify for metabolic syndrome and had a normal leucocyte count on admission, thereby strengthening the plausible relationship between hypertriglyceridemia and SSST.

The optimum treatment for CVST and its duration still remains uncertain, especially in the background of an unidentifiable etiology. ${ }^{10}$ On the basis of the limited available evidence at our disposal, anticoagulation appears to be safe and probably reduces the risk of death or dependency. ${ }^{11}$ The duration of anticoagulant therapy in CSVT depends on the etiology as well. In the presence of transient risk factors, anticoagulant therapy is given for three to six months whereas those with unknown causes 
or with hereditary thrombophilia are anticoagulated for longer duration due to high chances of recurrence. ${ }^{12}$

Our case might be considered among the $65-80 \%$ of CVST cases with one or more identifiable causes. Here, the main cause being elevated triglyceridemia which may have played a role in increasing our patient's thrombotic risk. This highlights a potential direct association between CVST and hypertriglyceridemia in the absence of other pre-disposing factors. If such a relationship between hypertriglyceridemia and CVST were to be found, new diagnostic measures might be warranted and this may also have an implication on the risk of recurrence and duration of anticoagulation in this group of patients.

\section{CONCLUSION}

Severe hypertriglyceridemia may trigger CVST. In this rare case study, hypertriglyceridemia was the only predisposing risk factor for developing CVST. Our case highlights the need for clinicians to consider CVST among patients with uncontrolled hypertriglyceridemia. New diagnostic measures may become necessary which may also have an implication on the risk of recurrence and duration of anticoagulant therapy in this group of patients.

\section{Acknowledgement}

The authors thank Mr. Kingshuk Bhattacharjee for the medical writing assistance in the development of this manuscript.

\section{References}

1. Stam J. Thrombosis of the cerebral veins and sinuses. New Engl J Med.2005; 352:1791-98. http://dx.doi.org/10.1056/NEJMra042354.

2. Bousser MG, Chiras J, Bories J, et al. Cerebral venous thrombosis - a review of 38 cases. Stroke.1985; 16:199-213. http://dx.doi.org/10.1161/01.STR.16.2.199.
3. Allroggen $\mathrm{H}$, Abbott RJ. Cerebral venous sinus thrombosis. Postgrad Med J. 2000; 76:12-15.

http://dx.doi.org/10.1136/pmj.76.891.12.

4. Kamphuisen PW, Eikenboom JC, vos HL, et al. Increased levels of factor VIII and fibrinogen in patients with venous thrombosis are not caused by acute phase reactions. ThrombHaemost. 1999; 81(5):680-683.

5. T-Y. Wang, J Yen, G-Y Hung, M-Y Hsieh, R-B Tang. A rare complication in a child undergoing chemotherapy for acute lymphoblastic leukemia: Superior sagittal sinus thrombosis. Journal of the Chinese Medical Association. 2011; 74:183-187. http://dx.doi.org/10.1016/j.jcma.2011.01.041.

6. Doggen CJM, Smith NL, Lemaitre RN, Heckbert SR, Rosendaal FR, Psaty BM. Serum lipid levels and the risk of venous thrombosis. Arterioscler Thromb Vasc Biol. 2004;24:1970 -1975. http://dx.doi.org/10.1161/01.ATV.0000143134.87051.46.

7. Candelaria GTB, Belangero VMS. "Predisposing factors for deep venous thrombosis in children and adolescents with nephrotic syndrome," ISRN Vascular Medicine. 2011; 1-5. http://dx.doi.org/10.5402/2011/827483.

8. Saxena R, Batra V, Singh ND, "Prothrombotic factors in nephrotic syndrome." Indian Journal of Pathology and Microbiology. 2000; 43(3)319-323

9. García-Raso A, Santos Montero AB, Ene GS, Llamas Sillero P. Cardiovascular risk factors and venous thromboembolism: Is there a connection? J Hematol Transfus. 2013; 1(2): 1009.

10. Ciccone A, Canhão P, Falcão F, et al. Thrombolysis for cerebral vein and dural sinus thrombosis. Cochrane Database Syst Rev. 2004; (1):CD003693.

11. de Bruijn SFTM, Stam J, for the Cerebral Venous Thrombosis Study Group. Randomised, placebo-controlled trial of anticoagulation treatment with low-molecular-weight heparin for cerebral sinus thrombosis. Stroke. 1999; 30: 484-488. http://dx.doi.org/10.1161/01.STR.30.3.484.

12. van Nuenen BFL, Munneke M, Bloem BR. Cerebral venous sinus thrombosis: Prevention of recurrent thromboembolism. Stroke. 2005; 36: 1822. http://dx.doi.org/10.1161/01.STR.0000176582.17934.82.

13. Mertens I, Verrijken A. Michiels JJ, et al. Among inflammation and coagulation markers, PAI- 1 is a true component of the metabolic syndrome. International Journal of Obesity. 2006; 30: 1308-1314. http://dx.doi.org/10.1038/sj.ijo.0803189.

Articles and any other material published in the JAFES represent the work of the author(s) and should not be construed to reflect the opinions of the Editors or the Publisher. Authors are required to accomplish, sign and submit scanned copies of the JAFES Declaration: that the article represents original material, that is not being considered for publication or has not been published or accepted for publication elsewhere. Consent forms, as appropriate, have been secured for the publication of information about patients; otherwise, authors declared that all means have been exhausted for securing such consent. The authors have signed disclosures that there are no financial or other relationships that might lead to a conflict of interest. All authors are required to submit Authorship Certifications that the manuscript has been read and approved by all authors, and that the requirements for authorship have been met by each author. 